Check for updates

Cite this: RSC Adv., 2018, 8, 20477

Received 8th May 2018

Accepted 29th May 2018

DOI: $10.1039 / \mathrm{c} 8 \mathrm{ra03928c}$

rsc.li/rsc-advances

\section{Quantum confined two-dimensional electron/hole gas switching by facet orientation of perovskite oxides $\dagger$}

\author{
Fei Zhou, ${ }^{\text {ab }}$ Yong Liu, ${ }^{\text {ad }}$ Zhonghong Lai, ${ }^{e}$ Mingqing Liao, (D) ${ }^{a}$ Yi Zhou, (D) a \\ Yudong Huang ${ }^{\mathrm{b}}$ and Jingchuan Zhu (iD) *acd
}

\section{Introduction}

Two-dimensional electron/hole gases (2DEGs/2DHGs) were first studied at the interfaces of semiconductor heterostructures..$^{1,2}$ Recent studies have revealed the existence of high mobility 2DEGs at $\mathrm{LaAlO}_{3} / \mathrm{SrTiO}_{3}$ interface ${ }^{3}$ and even at the bare surface of $\mathrm{SrTiO}_{3}{ }^{4,5}$ which are treated as the cornerstone of oxide electronics. ${ }^{6}$ Electronic reconstruction at interfaces or surfaces that occur to compensate for the polar discontinuity of adjacent constituents plays a key role in the formation of localized 2DEGs. ${ }^{7}$ This gives rise to attractive physics, such as insulatingmetallic state transitions, ${ }^{8}$ superconductivity, ${ }^{9}$ spiral magnetism and giant negative magnetoresistance. ${ }^{10-12}$ These above-

${ }^{a}$ School of Materials Science and Engineering, Harbin Institute of Technology, 150001, Harbin, China.E-mail:fgms@hit.edu.cn

${ }^{b}$ MIIT Key Laboratory of Critical Materials Technology for New Energy Conversion and Storage, School of Chemistry and Chemical Engineering, Harbin Institute of Technology, 150001, Harbin, China

'National Key Laboratory of Science and Technology on Advanced Composites in Special Environments, Harbin Institute of Technology, 150001, Harbin, China

${ }^{d}$ National Key Laboratory for Precision Hot Processing of Metals, Harbin Institute of Technology, 150001, Harbin, China

${ }^{e}$ Analysis and Testing Center, Harbin Institute of Technology, 150001, Harbin, China $\dagger$ Electronic supplementary information (ESI) available: Computation methods, surface slabs of different facets of $\mathrm{KNbO}_{3}$, band structure of trilayers models of $2 \mathrm{D} \mathrm{KNbO}{ }_{3}$, crystal models of $(100)_{\text {orth }}$ oriented $2 \mathrm{D} \mathrm{KNbO}_{3}$, band structures of (100) orth oriented $2 \mathrm{D}$ crystal, states occupation below $E_{\mathrm{F}}$ of $(100)_{\text {orth }}$ oriented $2 \mathrm{D}$ crystals, free electrons absorptions of $(100)_{\text {orth }}$ oriented $2 \mathrm{D} \mathrm{KNbO}_{3}$, crystal models of $(120)_{\text {orth }}$ oriented 2D crystal, band structures of (120) orth oriented 2D crystal, electronic structures of bulk $\mathrm{KNbO}_{3}$ crystal (PDF). See DOI: $10.1039 / \mathrm{c} 8 \mathrm{ra03928c}$ mentioned examples occur on n-type interface/surface, such as the interface construction of $(\mathrm{LaO})^{+} /\left(\mathrm{TiO}_{2}\right)^{0}$ or with defects created on bare $\mathrm{SrTiO}_{3}$ surface, leading to the mixed dimensionality of confined conducting electrons. ${ }^{13}$ On the contrary, it is still challenging to obtain the free holes at interfaces or surfaces of oxides, ${ }^{3,14}$ although recent experiment shows that strong hole carriers can be seen at the $\mathrm{LaAlO}_{3} / \mathrm{SrTiO}_{3}$ heterointerface by reducing the oxygen defects. ${ }^{15,16}$ However, 2DHGs have been researched in $\mathrm{Ge} / \mathrm{Si}$ based semiconductor heterostructures, presenting novel physics, such as spin Hall effect induced edge-spin accumulation and well-controlled Coulomb blockade oscillations. ${ }^{17-19}$ The lack of investigations of 2DHGs on perovskite oxides might be ascribed to the limitation of current epitaxial growth technology, which generally allows films to grow along $(001)_{\text {cubic }}$ direction through substrate matching, preserving an extra $\mathrm{e}^{-} / 2$ per unit cell on the discontinued interface or surface. Our recent experiments show that $2 \mathrm{D}$ potassium niobates can be grown automatically in liquid with facets of $60^{\circ}$ tilting of oxygen octahedrons, providing a practical basis for the study of the facets effects to 2DEGs/ 2DHGs.

In this letter, $a b$ initio calculation is used to investigate the confined 2DEGs/2DHGs in $2 \mathrm{D} \mathrm{KNbO}_{3}$. Results show that the increase of polar discontinuity by facets engineering would change the electronic phase of the material surface, leading to the formation of switchable 2DEGs and 2DHGs on $2 \mathrm{D} \mathrm{KNbO}_{3}$ structures by varying facet orientations. These free electron/hole states around the Fermi level $\left(E_{\mathrm{F}}\right)$ are strongly quantum localized. This concept not only endows the material with an 
insulator-metal transition, but also provide an opportunity for manipulating 2DEGs/2DHGs in the quantum confined regime.

\section{Computational details}

The first-principle calculation is performed with the Cambridge Serial Total Energy Package. ${ }^{20} 2 \mathrm{D}$ crystal models are fully relaxed through geometry optimization according to system energy, force and stress by DFT with the Perdew, Burke, and Ernzerhof functional. ${ }^{21}$ For our main objective is qualitatively investigating the behaviors of quantum confined free electronic states, ultrasoft pseudopotentials are applied to describe the ionic cores with a plane-wave cutoff energy of $340 \mathrm{eV}$. The $k$-point sampling grids are $3 \times 3 \times 1$ for 2 D crystal slabs and $4 \times 4 \times 2$ for bulk $\mathrm{KNbO}_{3}$ crystal, respectively. The vacuum spacing layer between neighboring repeat units of $2 \mathrm{D}$ slabs are set to $1 \mathrm{~nm}$. The truncated surface are capped by $\mathrm{H}$ atoms, in order to neutralize the surface dangling oxygen bonds.

The surface energy $E_{\mathrm{s}}$ is computed using the formula $E_{\mathrm{s}}=$ $\left(E_{\text {slab }}-n E_{\text {bulk }}\right) / 2 A,{ }^{22}$ where $E_{\text {slab }}$ and $E_{\text {bulk }}$ are the total energy of the $2 \mathrm{D}$ slab and the bulk per unit cell, respectively. They are all obtained by DFT calculation. $n$ is the number of bulk unit cell contained in the $2 \mathrm{D}$ slab. $2 A$ is the surface area of each $2 \mathrm{D}$ slabs.

\section{Results and discussion}

\section{DEGs/2DHGs switching}

$\mathrm{KNbO}_{3}$ is a typical orthorhombic ferroelectric with $\mathrm{K}^{+}$and $\mathrm{Nb}^{5+}$, showing different valence discontinuity from conventional $\mathrm{SrTiO}_{3}$ in various facets orientation. In order to know the facets differences of orthorhombic $\mathrm{KNbO}_{3}$, density functional theory is used. Two dimensional (2D) slabs with tilted oxygen octahedrons in angles of $0^{\circ}, 60^{\circ}, 90^{\circ}$ are built (as sketched in Fig. 1, the $2 \mathrm{D}$ slabs used for DFT calculation see Fig. S1 $\dagger$ ). Calculated results show that the facets of $(100)_{\text {orth }},(011)_{\text {orth }}$ and $(01 \overline{1})_{\text {orth }}\left(0^{\circ}\right.$ tilting of oxygen octahedron, see Fig. 1a), which are perpendicular to one another with a terminal plane of $(\mathrm{KO})^{-}$, have small surface energy $\left(E_{\mathrm{s}}\right)$ below $100 \mathrm{MeV}^{-2}$ (see Table 1). Lower surface energies explains why $\mathrm{KNbO}_{3}$ crystals always grow as cubic particle in hydrostatic reactions. These facets correspond to the $(100)_{\text {cub }},(010)_{\text {cub }}$ and $(001)_{\text {cub }}$ oriented facets in cubic $\mathrm{SrTiO}_{3}$ respectively, but with distinct valence difference in alternate atomic layers, $(\mathrm{SrO})^{0} /\left(\mathrm{TiO}_{2}\right)^{0}$ in $\mathrm{SrTiO}_{3}$ and $(\mathrm{KO})^{-} /$ $\left(\mathrm{NbO}_{2}\right)^{+}$in $\mathrm{KNbO}_{3}$. Besides $0^{\circ}$ tilted octahedron 2D models, the $E_{\mathrm{s}}$ of $60^{\circ}$ and $90^{\circ}$ tilted models (Fig. $1 \mathrm{~b}$ and c) are all between $150 \mathrm{MeV} \AA^{-2}$ and $300 \mathrm{MeV} \AA^{-2}$, maintaining a strong energy barrier in self-driven growth processes. The large differences in surface energies will work on the formation of stable surface structures of $2 \mathrm{D} \mathrm{KNbO}_{3}$. This further results in their distinct electronic reconstructions on the surface.

To investigate the relevance of surface energy to surface polar properties, trilayers $2 \mathrm{D}$ models of $\mathrm{KNbO}_{3}$ crystal with $(100)_{\text {orth }}$ and $(120)_{\text {orth }}$ facet orientations are built. After lattice relaxation, stable 2D structures are obtained (Fig. 2a and c). Their electronic structures are calculated. Specifically, the $60^{\circ}$ tilted facet of $(120)_{\text {orth }}$ 2D crystal shows an alternating valence variation of $\left(\mathrm{KO}_{3}\right)^{5-} / \mathrm{Nb}^{5+}$, indicating a potential huge polar difference in alternating atomic planes, as shown in Fig. 2d. From the sliced mapping of electron localization functions (ELF) in Fig. 2b and d, quantum confined surface states can be clearly observed on both surfaces, which surround the perfectly localized core and the bonding electrons (where ELF approaches 1$)^{23}$ of oxygen atoms, allowing the electron and hole carriers moving freely on the surface. In consideration of the

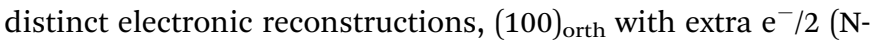
type) and $(120)_{\text {orth }}$ with extra $3 \mathrm{~h}^{+} / 2$ (P-type) injection per unit cell, their electronic phases should be different. Fig. 3a and b show the calculated bands around $E_{\mathrm{F}}$ of (100) orth and (120) orth oriented $2 \mathrm{D} \mathrm{KNbO}_{3}$. Conventional free electron conductance switches to free hole conductance when a facet of $(100)_{\text {orth }}$ changes to $(120)_{\text {orth }}$ by the $60^{\circ}$ tilting of oxygen octahedrons. The preferential growth facet also changes the symmetry of electronic states transitions, resulting in an indirect optical band gap transition changing to direct at $\Gamma$ point, as the red arrows indicated in ESI Fig. S2a and S2b. $\dagger$ Density of states of s, p, d orbits (Fig. 3c and d) shows further insights of this switching behavior. The potential of low-lying conduction band descends by $0.39 \mathrm{eV}$ due to extra electron injection, pushing the $\mathrm{Nb} 4 \mathrm{~d}$ band to $E_{\mathrm{F}}$ and allowing electrons to move freely at the bottom of the conduction band (Fig. 3c). Conversely, extra holes injection induced by strongly quantized $\mathrm{O} 2 \mathrm{p}$ states lifts the valence band by $0.17 \mathrm{eV}$ above $E_{\mathrm{F}}$, giving free holes. The low-
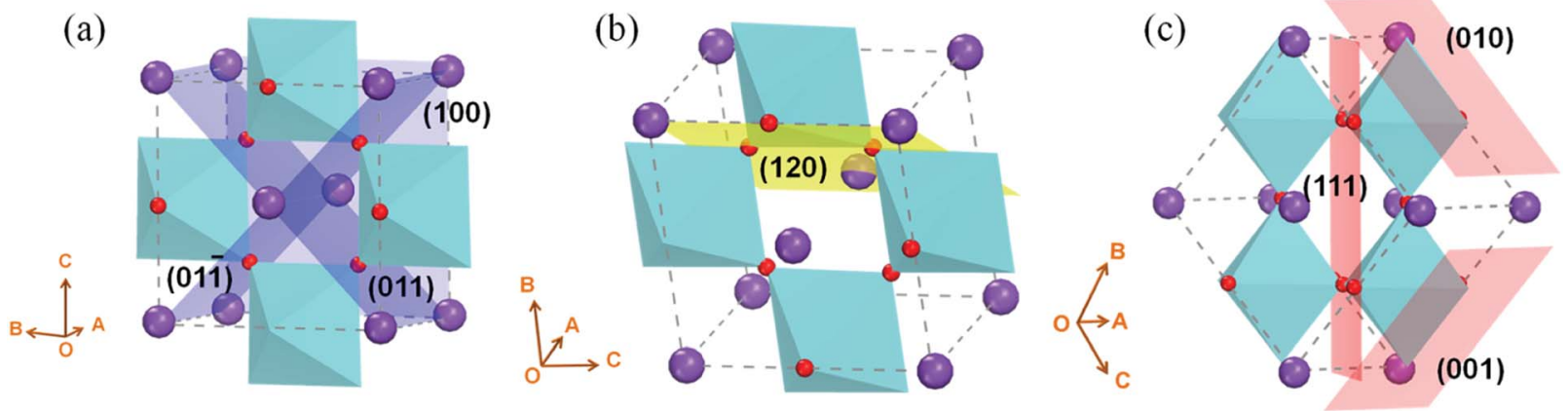

Fig. 1 (a) (100), (011) and (011) facets of $A m m 2$ orthorhombic $\mathrm{KNbO}_{3}$ (cut plane marked by blue) with $0^{\circ}$ tilt of oxygen octahedrons, these three facets are perpendicular to each other, forming a cubic morphology. (b) (120) facet (cut plane marked by yellow) with $60^{\circ}$ tilt of oxygen octahedrons, shows a pseudo-hexagonal symmetry in plane. (c) (001), (010) and (111) facets (cut plane marked by red) with $90^{\circ}$ tilt of oxygen octahedrons. 
Table 1 The energy difference of 2D slab to bulk $\left(E_{\text {slab }}-E_{\text {bulk }}\right)$, surface areas $(S)$, surface energy $\left(E_{s}\right)$ of $2 \mathrm{D} \mathrm{KNbO}_{3}$ with different facets

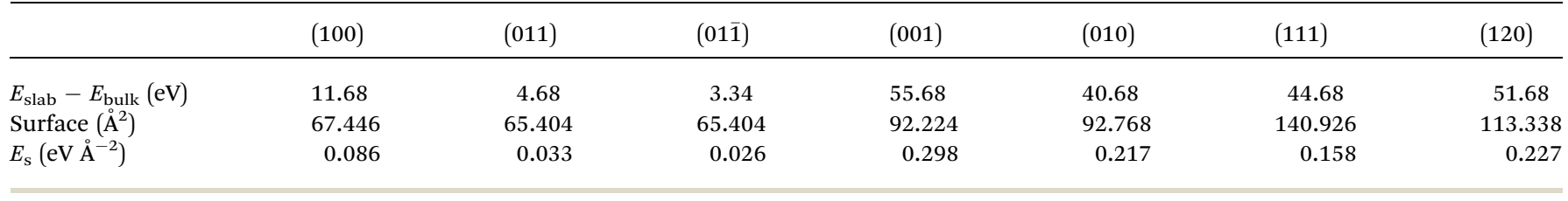

lying subbands of $\mathrm{Nb} 4 \mathrm{~d}$ are split $\mathrm{t}_{2 \mathrm{~g}}$ bands, composed of a heavy $\left(\mathrm{d}_{y z}\right.$ doublet) and a light $\left(\mathrm{d}_{x y}\right.$ singlet) band. ${ }^{24}$ Strong confinement lifts up the light $t_{2 \mathrm{~g}}$ band with respect to the heavy ones (Fig. 3a), demonstrated in the thickness dependence of

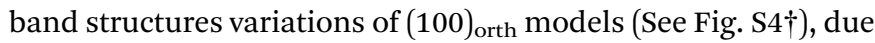
to the difference in $m_{\mathrm{e}}$. The top $\mathrm{O} 2 \mathrm{p}$ bands also split into three subbands, the highest heavy $\left(E_{\mathrm{hb}}{ }^{+}\right)$and underlying two light $\left(E_{\mathrm{lb}}{ }^{+}\right)$ones (Fig. 3b) under the quantum confined regime.

\section{Quantized electronic states in a 2D square quantum well}

To obtain the effect of the size limitation to free electronic states, the band structures of $(120)_{\text {orth }} 2 \mathrm{D} \mathrm{KNbO}_{3}$ with thicknesses that range from $0.23 \mathrm{~nm}$ (1 octahedron layer) to $4.6 \mathrm{~nm}$ (20 octahedron layers) are calculated (see Fig. S8†). Strong quantum confinement can be observed with a decrease in thickness, accompanied by the splitting of degenerate energy into independent sub-bands, as indicated by the red arrow in ESI Fig. S8. $\dagger$ A two-dimensional square quantum well is used to describe the thickness dependence of quantum confined band gaps, given by: $E_{\mathrm{g}}=E_{\mathrm{g}}^{0}+\frac{h^{2} \pi^{2}}{2 m^{*} r^{2}},{ }^{25}$ where $E_{\mathrm{g}}$ is the calculated energy gap of the 2D model and $E_{\mathrm{g}}^{0}$ is the calculated energy gap of the bulk crystal model $\left(E_{\mathrm{g}}^{0} \sim 2.3 \mathrm{eV}\right.$, as shown in ESI Fig. S9†). The second term represents quantum localization, where $\hbar$ is the reduced Planck's constant, $r$ is the sheet thickness, and $m^{*}=\left(\frac{1}{m_{\mathrm{e}}}+\frac{1}{m_{\mathrm{h}}}\right)^{-1}$ is the reduced effective mass. Fig. 4a plots the calculated thickness dependence of quantum well transition energies with dimension reduction. The value of $m^{*}$ is 0.6 $m_{\mathrm{e}}$, which is obtained from fitting a best fit line to calculate $E_{\mathrm{g}}$ values (pink dotted line in Fig. 4a). The solid fit line in the inset confirms the linear relationship of quantum confined energies to $1 / r^{2}$ for a $2 \mathrm{D}$ square quantum well.

The hole states occupation of samples are shown in Fig. 4bh. A monotonous decreasing relationship of confined energy of hole states with respect to the thicknesses of $2 \mathrm{D}$ wells is observed, where the $\mathrm{O} 2 \mathrm{p}$ bands will be totally under $E_{\mathrm{F}}$ when thickness approaches $4.6 \mathrm{~nm}$. Strong confinement leads to the splitting of the top O 2p band into one heavy $\left(E_{\mathrm{hb}}{ }^{+}\right.$, denoted as red dots) and two light $\left(E_{\mathrm{lb} 1}{ }^{+}\right.$and $E_{\mathrm{lb} 2}{ }^{+}$, denoted as blue and green dots respectively) bands at $\Gamma$ point. The decrease in quantum confined energy contributes to the appearance of degenerate $E_{\mathrm{hb}}{ }^{+}$and $E_{\mathrm{lb} 1}{ }^{+}$bands (as shown in Fig. $4 \mathrm{~b}-\mathrm{f}$ ), accompanied by the gradual increase of $m_{\mathrm{h}}$ of $E_{\mathrm{lb} 1}{ }^{+}$along momentum $k$. Until reaching the weak confined situation (Fig. 4g), the light $E_{\mathrm{lb} 2}{ }^{+}$band descends down to $E_{\mathrm{F}}$ first, then degenerate $E_{\mathrm{hb}}{ }^{+}$and $E_{\mathrm{lb} 1}{ }^{+}$bands will further combine with $E_{\mathrm{lb} 2}{ }^{+}$ and totally descend under $E_{\mathrm{F}}$ (Fig. $4 \mathrm{~h}$ ). A similar trend of lowlying conduction $t_{2 g}$ band of $(100)_{\text {orth }}$ models being lifted up to $E_{\mathrm{F}}$ can be observed with an increase in thickness (see ESI (a) $(100)_{\text {orth }}$

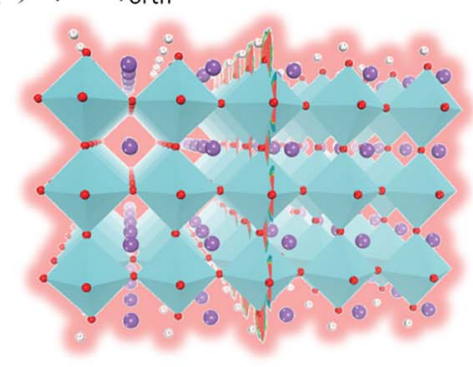

(c) $(120)_{\text {orth }}$

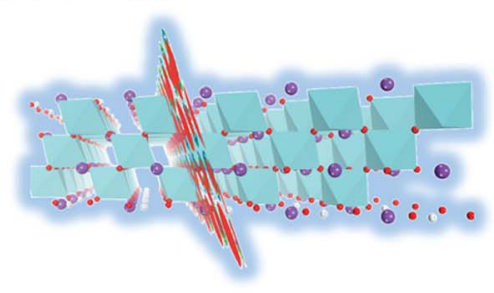

(b)

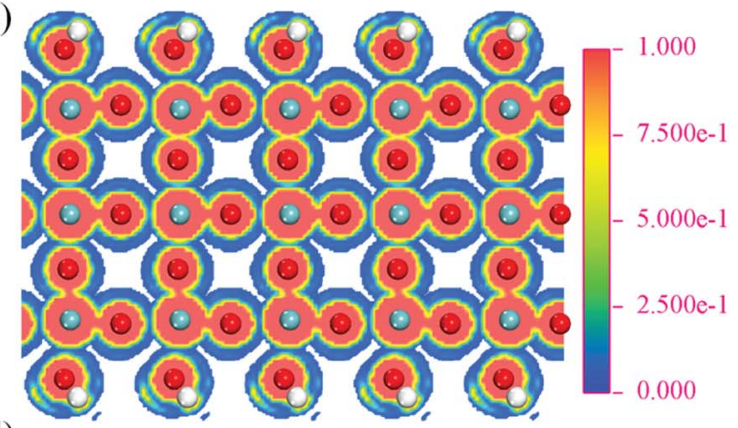

(d)
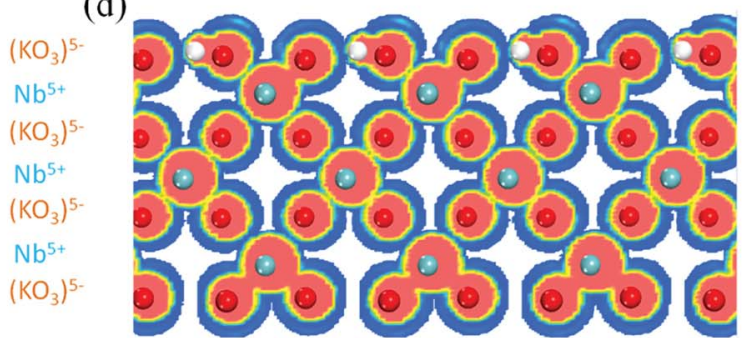

Fig. 2 (a) and (c) The trilayers (octahedron layer) atomic structures of (100) orth and (120) orth oriented 2D $\mathrm{KNbO}_{3}$ models, surface dangling bonds of oxygen were capped by hydrogen atoms, the selected cutting slices of Nb-O plane of calculated ELF in (a) and (c) were showed in (b) and (d) by colored maps, respectively. Both of the 2D structures were obtained by energy minimization through optimizing geometry. 

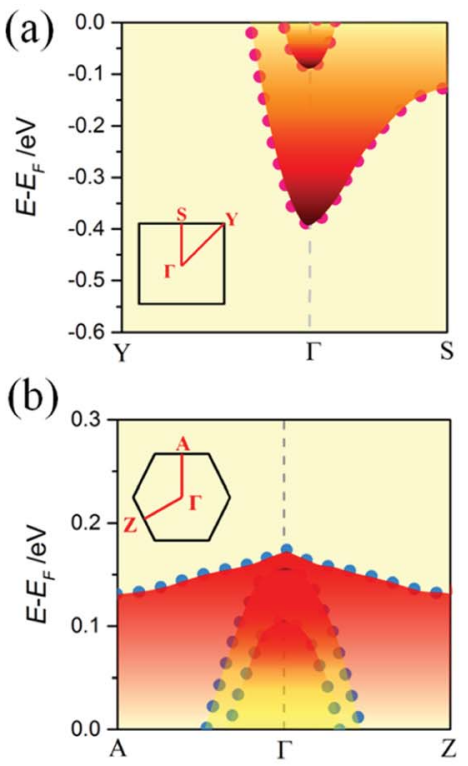

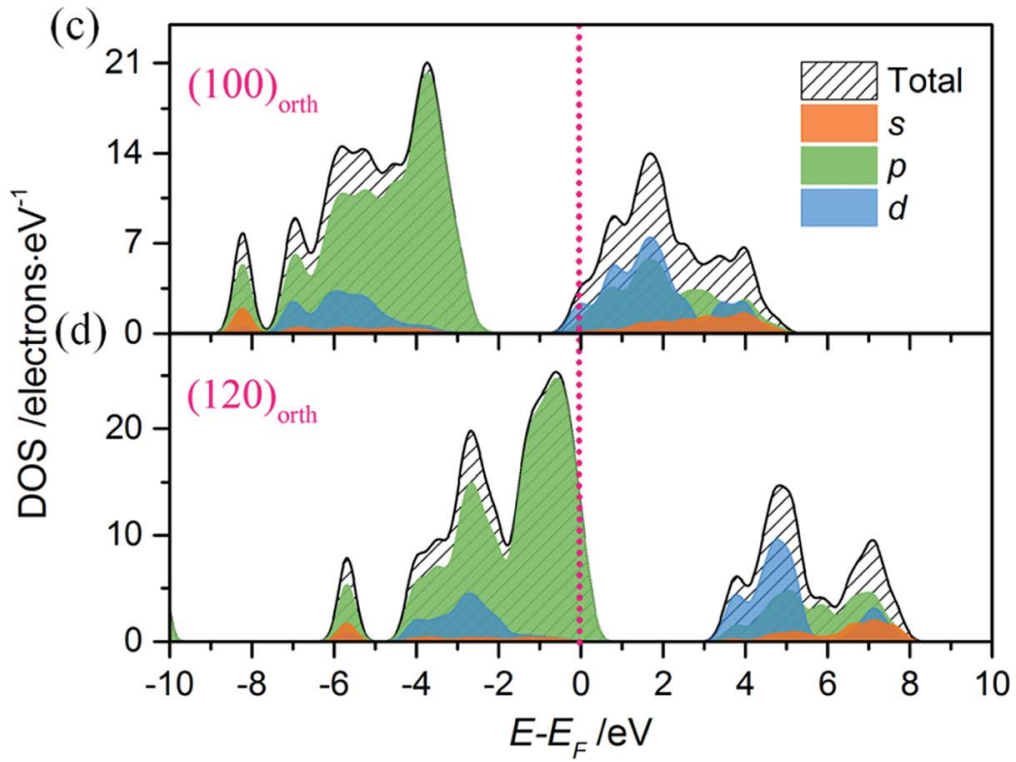

Fig. 3 (a) and (b) Calculated band structures of trilayers (octahedron layer) (100) orth and (120) orth oriented 2D KNbO 3 models, respectively. Free electron states switch to free holes when facets changed. (c) and (d) Partial density of states of s, p, d orbits of both of $2 \mathrm{D}$ KNbO 3 models, respectively. Oblique fill lines show the sum of DOS of different orbits.

Fig. S5†), showing that both of 2 DEG and 2 DHG cases have strong quantum confinement along the $Z$ direction.

\section{Insulator-metal transition}

To obtain further insight of the insulator-metal transition of our 2DFEG/2DFHG systems, optical absorption properties of models with varying thicknesses are calculated. Fig. 5a shows the absorption spectra of $(120)$ orth oriented $2 \mathrm{D} \mathrm{KNbO}_{3}$ models (thicknesses are from $0.46 \mathrm{~nm}$ to $4.6 \mathrm{~nm}$ ). The strong absorption across the whole optical gap demonstrates the insulatormetal transition. Fig. 5b plots the maximum absorption of the free hole states (collected from low-frequency absorption spectra, as shown in inset figure), showing that the free holes absorption intensity increase gradually with an increase in thickness. Up to above $1.15 \mathrm{~nm}$ (5 layers), it decreased dramatically with an increase in thickness, indicating a critical thickness of about $2 \mathrm{~nm}$ for the existence of metallic states for 2DHGs. A gradual blue-shift of intrinsic absorption band edges can be observed when the number of layers increases (indicated by the blue arrow in Fig. 5a), along with one single peak extending to two individual peaks (as pink arrow indicated in Fig. 5a) due to the splitting and lifting of s, p orbits from the (a)

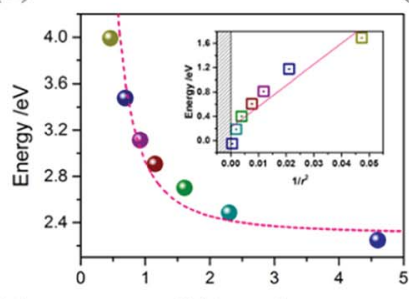

(e)

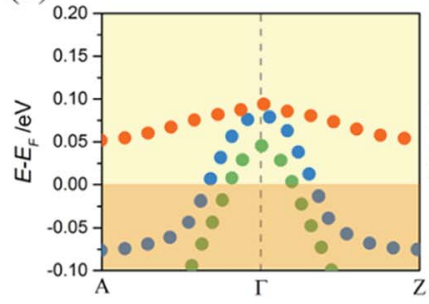

(b)

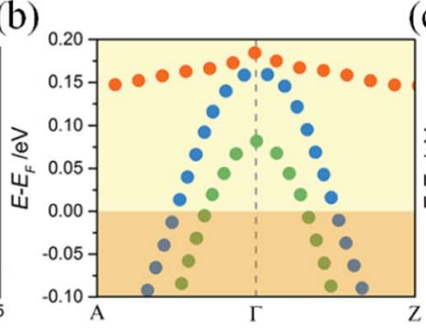

(f)

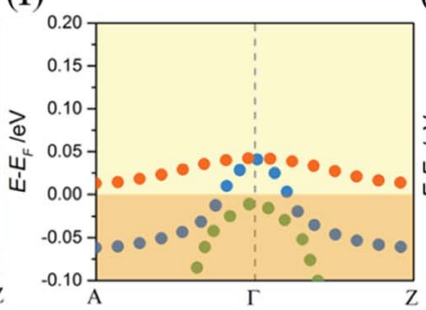

(c)

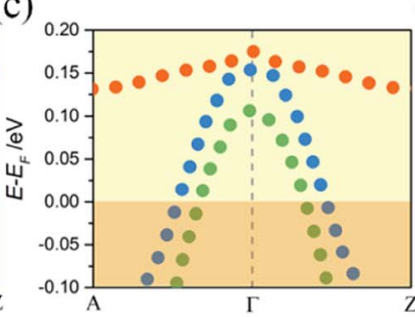

(g)

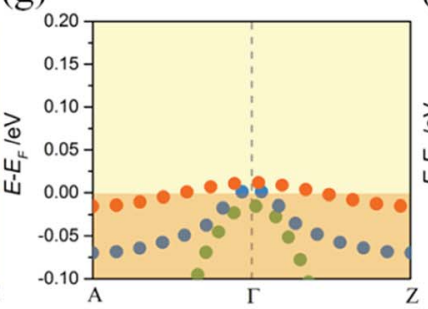

(d)

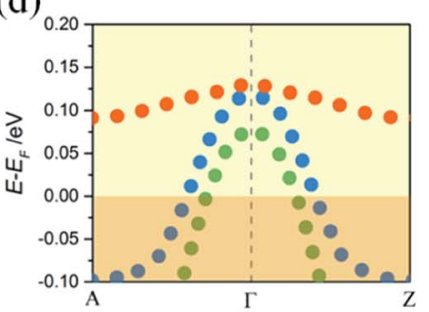

(h)

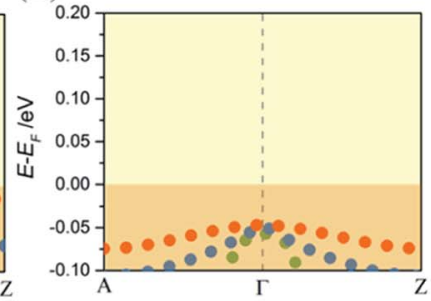

Fig. 4 (a) Colored balls correspond to the calculated band gap energy of 2D models with thicknesses varying from $0.46 \mathrm{~nm}$ to $4.6 \mathrm{~nm}$. The abrupt increase in band gap energy occurs at below 2 nanometer size, where there is a presence of strong quantum confinement. The theoretically calculated curve (pink dotted line) fits very well to the experimental data. Inset illustrates the linear relationship between the quantum confinement effected energy values and $1 / r_{2}$. (b)-(h) The evolution of quantum localized hole states with $2 \mathrm{D}$ well thickness varying. Red dotted line denotes the heavy hole $\left(E_{\mathrm{hb}}{ }^{+}\right)$band, blue and green dotted lines denote the two light $\left(E_{\mathrm{lb} 1}{ }^{+}\right.$and $\left.E_{\mathrm{lb} 2}{ }^{+}\right)$bands. 
(a)

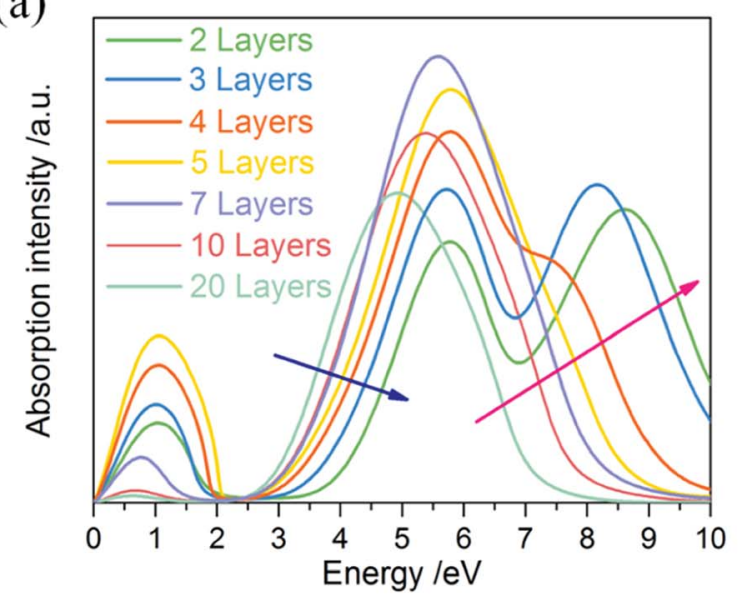

(c)

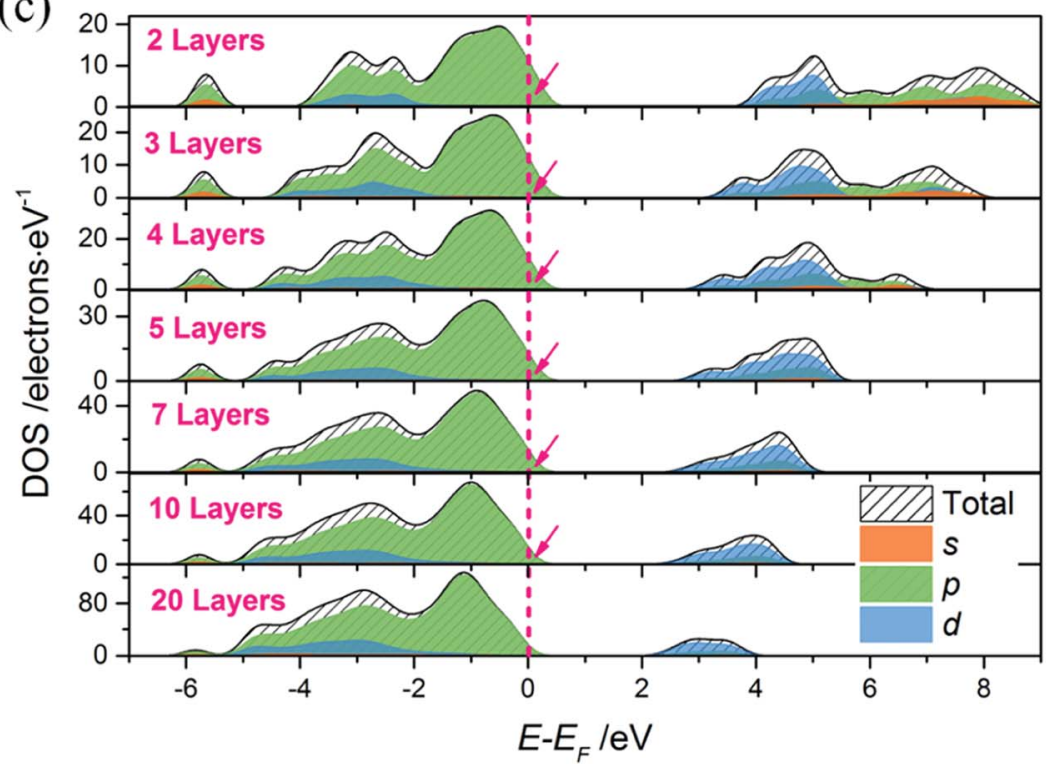

(b)

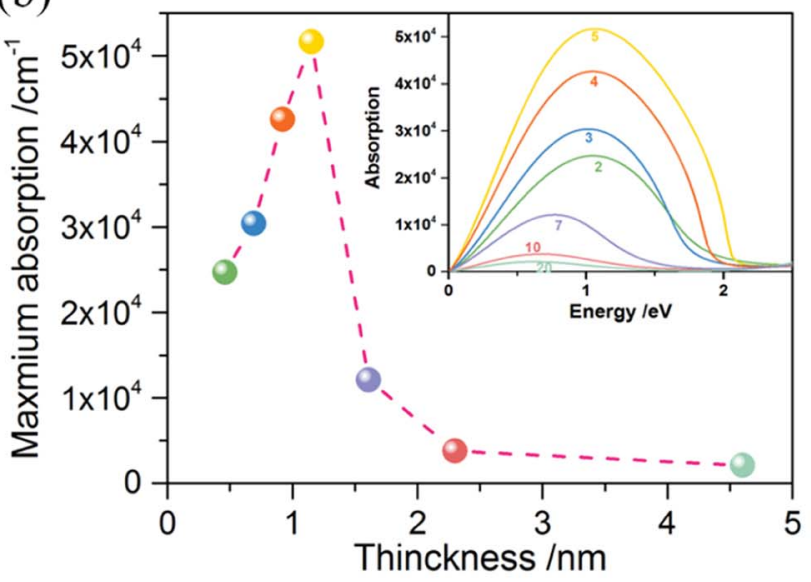

(d)

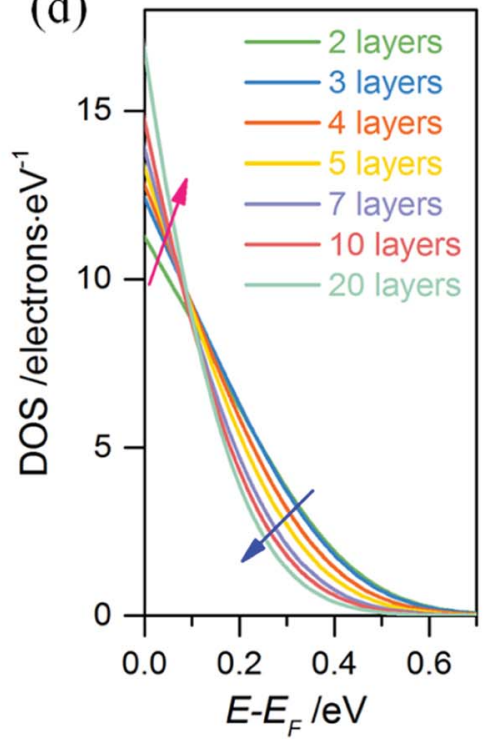

Fig. 5 (a) Absorption properties of (120) orth oriented $2 \mathrm{D} \mathrm{KNbO}_{3}$ models, the absorption in low frequency indicates an insulator-metal transition absorption. (b) The thickness dependences of absolute absorption intensity of free hole states, inset is the zoom in of low-frequency range. (c) PDOS of (120) orth oriented $2 \mathrm{D} \mathrm{KNbO}_{3}$ with thickness range from 2 layers to 20 layers, show the thickness dependence of quantum localized free holes and the corresponding contributions of different orbits. (d) Evolution trend of quantized hole states with respect to the thickness varying. The quantum localized energy and the absolute value of electronic states per electron volt co-work on the free hole states absorption, showing a converse contribution (as the blue and pink arrows indicating with reverse directions).

degenerate $\mathrm{d}$ band (Fig. 5c). This agrees with the quantized characters of conduction band in 2D quantum well. Although the occupation energy of hole states above $E_{\mathrm{F}}$ decreases with an increase in well thickness (such as the pink arrow marked in Fig. $5 \mathrm{c}$ and blue arrow marked in Fig. $5 \mathrm{~d}$ ), the increase of DOS near $E_{\mathrm{F}}$ (marked by pink arrow in Fig. 5d) competes with the decrease of confined energy, leading to an increase initially then decrease of free hole states absorptions. The increase in DOS near $E_{\mathrm{F}}$ is ascribed to the increased total numbers of atoms per unit area when stacking layers increase. However, the confined energy still plays a dominant role for metallic states maintaining, as plotted in Fig. 4b. The absorption properties of free electrons ((100) orth oriented $2 \mathrm{D} \mathrm{KNbO}_{3}$ models) show a similar trend of confined states transition while the thickness variation (see ESI Fig. S6a $\uparrow$ ), although the $\sim 4 \mathrm{~nm}$ (10 layers) model shows an anomalous absorption in the low-frequency range, which can be ascribe to the abrupt increase of the local DOS near $E_{\mathrm{F}}$, as marked by pink arrow in ESI Fig. S6b. $\dagger$

\section{Conclusions}

In summary, beyond well-known concepts for rearranging these surface electronic states (with extra electrons injecting) in 2D heterointerface or bare surface, our results show that additional holes injection can be maintained by the increase of surface polarity. The 2DEGs/2DHGs switching can be simply fulfilled by facet orientations. Distinct orientations change the polar discontinuity of alternating atom layers, corresponding to that huge surface energy differences. The thickness dependent behaviors of free electron/hole states subjecting to quantum confinement are addressed by the varied thickness of $2 \mathrm{D}$ 
models. Compared with free electron states induced insulatormetal transition (4 $\mathrm{nm}$ sample still exists strong free electron states absorption), free hole states transition depends much stronger on the thickness of $2 \mathrm{D}$ quantum well, with a small critical value of $\sim 2 \mathrm{~nm}$. The formation of free electrons is more complex than free holes (contributed by $\mathrm{O} 2 \mathrm{p}$ states) due to the hybridization of $\mathrm{s}, \mathrm{p}, \mathrm{d}$ orbits in conduction bands. Such as one $\mathrm{p}$ band abnormally extends under $E_{\mathrm{F}}$ (among the split $\mathrm{t}_{2 \mathrm{~g}}$ bands) for $(100)_{\text {orth }}$ oriented monolayer model at the extreme confined regime, acting to form an additional free electron conduction band (see ESI Fig. S5a $\dagger$ ). Despite their differences, both of 2DEGs and 2DHGs are resulted from strong quantum confinement. Furthermore, the confined energy $\left(E-E_{\mathrm{F}}\right)$ and the total DOS occupying near $E_{\mathrm{F}}$ (which varies with sample thickness) jointly act on the metallic state transition, demonstrating that metallic states can be modulated not only by the amount of donor-like defects or adsorbates, but also simply by thickness variation.

In contrast to previous theoretical and experimental results, ${ }^{\mathbf{3 , 4 , 2 4 , 2 6}}$ our theoretical study reveals that the free holes can be obtained on high polar surface of perovskite oxides, gives the origins of oxides 2DEGs/2DHGs, including their intrinsic switching by surface polarity change and confined evolution behaviors with respect to quantum well thickness, emerging as a promising basis towards design the new generation all-oxides electronic devices.

\section{Conflicts of interest}

There are no conflicts to declare.

\section{Acknowledgements}

The work at HIT is supported by the Fundamental Research Funds for the Central Universities. Grant No. Hit. KISTP. 201401.

\section{References}

1 E. E. Mendez, L. Esaki and L. L. Chang, Phys. Rev. Lett., 1985, 55, 2216.

2 J. M. Kikkawa, Science, 1997, 277, 1284-1287.

3 A. Ohtomo and H. Y. Hwang, Nature, 2004, 427, 423-426.

4 W. Meevasana, P. D. C. King, R. H. He, S.-K. Mo, M. Hashimoto, A. Tamai, P. Songsiriritthigul, F. Baumberger and Z.-X. Shen, Nat. Mater., 2011, 10, 114118.

5 A. F. Santander-Syro, O. Copie, T. Kondo, F. Fortuna, S. Pailhes, R. Weht, X. G. Qiu, F. Bertran, A. Nicolaou, A. Taleb-Ibrahimi, P. Le Fevre, G. Herranz, M. Bibes, N. Reyren, Y. Apertet, P. Lecoeur, A. Barthelemy and M. J. Rozenberg, Nature, 2011, 469, 189-193.
6 C. Cen, S. Thiel, J. Mannhart and J. Levy, Science, 2009, 323, 1026-1030.

7 S. Okamoto and A. J. Millis, Nature, 2004, 428, 630-633.

8 C. Cen, S. Thiel, G. Hammerl, C. W. Schneider, K. E. Andersen, C. S. Hellberg, J. Mannhart and J. Levy, Nat. Mater., 2008, 7, 298-302.

9 N. Reyren, S. Thiel, A. D. Caviglia, L. F. Kourkoutis, G. Hammerl, C. Richter, C. W. Schneider, T. Kopp, A. S. Ruetschi, D. Jaccard, M. Gabay, D. A. Muller, J. M. Triscone and J. Mannhart, Science, 2007, 317, 11961199.

10 A. Brinkman, M. Huijben, Z. M. Van, J. Huijben, U. Zeitler, J. C. Maan, V. D. W. Wg, G. Rijnders, D. H. Blank and H. Hilgenkamp, Nat. Mater., 2007, 6, 493-496.

11 S. Banerjee, O. Erten and M. Randeria, Nat. Phys., 2013, 9, 625-629.

12 A. F. Santander-Syro, F. Fortuna, C. Bareille, T. C. Rödel, G. Landolt, N. C. Plumb, J. H. Dil and M. Radović, Nat. Mater., 2014, 13, 1085.

13 N. C. Plumb, M. Salluzzo, E. Razzoli, M. Månsson, M. Falub, J. Krempasky, C. E. Matt, J. Chang, M. Schulte and J. Braun, Phys. Rev. Lett., 2014, 113, 086801.

14 M. Huijben, G. Rijnders, D. H. Blank, S. Bals, S. Van Aert, J. Verbeeck, G. Van Tendeloo, A. Brinkman and H. Hilgenkamp, Nat. Mater., 2006, 5, 556-560.

15 Y. Chen and N. Pryds, Nat. Mater., 2018, 17, 215-216.

16 H. Lee, N. Campbell, J. Lee, T. J. Asel, T. R. Paudel, H. Zhou, J. W. Lee, B. Noesges, J. Seo and B. Park, Nat. Mater., 2018, 17, 231-236.

17 W. Lu, J. Xiang, B. P. Timko, Y. Wu and C. M. Lieber, Proc. Natl. Acad. Sci. U. S. A., 2005, 102, 10046.

18 K. Nomura, J. Wunderlich, J. Sinova, B. Kaestner, A. H. MacDonald and T. Jungwirth, Phys. Rev. B, 2005, 72, 245330.

19 R. Moriya, K. Sawano, Y. Hoshi, S. Masubuchi, Y. Shiraki, A. Wild, C. Neumann, G. Abstreiter, D. Bougeard and T. Koga, Phys. Rev. Lett., 2014, 113, 2916-2921.

20 M. D. Segall, P. J. D. Lindan, M. J. Probert, C. J. Pickard, P. J. Hasnip, S. J. Clark and M. C. Payne, J. Phys.: Condens. Matter, 2002, 14, 2717.

21 J. P. Perdew, K. Burke and M. Ernzerhof, Phys. Rev. Lett., 1996, 77, 3865-3868.

22 Y. Bi, S. Ouyang, N. Umezawa, J. Cao and J. Ye, J. Am. Chem. Soc., 2011, 133, 6490-6492.

23 B. Silvi and A. Savin, Nature, 1994, 371, 683-686.

24 P. Delugas, A. Filippetti, V. Fiorentini, D. I. Bilc, D. Fontaine and P. Ghosez, Phys. Rev. Lett., 2011, 106, 166807.

25 L. E. Brus, J. Chem. Phys., 1984, 80, 4403-4409.

26 Z. S. Popović, S. Satpathy and R. M. Martin, Phys. Rev. Lett., 2008, 101, 256801. 